\title{
A modified concept for carrying out and interpretation of multi-rate gas well deliverability testing, using flow rate control choke
}

\begin{abstract}
This paper presents a modified procedure for the execution and interpretation of results for multi-rate gas well deliverability testing. The proposed procedure requires using a flow regulation device, which allows for the control of the gas flow rate. The exponent in conventional gas well deliverability equation is allowed to be different from 2 . Provided are example interpretations of the multi-rate gas wells deliverability tests carried out according to the proposed procedure using the real world data.
\end{abstract}

Key words: multi-rate production test, laminar/turbulent flow coefficients, deliverability, absolute open flow potential.

\section{Zmodyfikowany sposób przeprowadzania i interpretacji wyników wielocyklowego testu produkcyjnego odwiertu gazowego}

Przedstawiono zmodyfikowaną metodykę realizacji i interpretacji wyników wielocyklowego testu przypływu do odwiertu gazowego. Do przeprowadzenia testowań odwiertu gazowego zgodnie z proponowaną metodyką wymagane jest użycie zwężki o regulowanym przelocie umożliwiającej uzyskanie żądanego natężenia przepływu gazu. Dopuszczono różną od 2 wielkość wykładnika potęgowego w tzw. formule dwuczłonowej. Załączono przykłady obliczeniowe dla odwiertów gazowych.

Słowa kluczowe: test produkcyjny wielocyklowy, współczynniki formuły dwuczłonowej, potencjalne natężenie wypływu gazu.

\section{Introduction}

The conventional multi-rate gas well test consists of the production of the well at various stabilized flow rates and measuring the stabilized sand face pressure at the end of each flow cycle. After each flow cycle is completed, the well is closed for pressure stabilization until pressure builds up to its original value which is equal to the average pressure within the drainage area of the well being tested. The aim of gas well testing is to measure the production capabilities at the specific conditions of a reservoir and bottom hole flowing pressure. The multi-rate gas well test enables calculation of the maximum flow potential of the well $\left(Q_{a b s}\right)$ and generates the inflow performance curve, which indicates the relation between surface flow rate and bottom hole flowing pressure for a given value of average pressure, within the drainage area of the well.
Besides conventional multi-rate gas well tests for which build up periods are continued until stabilization of pressure is reached, there are several other deliverability testing methods developed to shorten the testing time including:

- Flow after flow tests, which consist of flowing the well at a series of constant flow rates and measuring the stabilized sand face pressure; the flowing periods are not followed by pressure build up periods so the final flow pressure of the preceding cycle is the initial pressure of the next flow cycle.

- An isochronal test, which shortens testing time by skipping pressure stabilization for flow cycles, while build up pressure periods are continued until the pressure is stabilized, to the average pressure within the drainage area. 
- A modified isochronal test in which the flow periods are of equal duration. Likewise, the pressure build up periods are also of equal duration, but not necessarily the same as the flow periods.

The equation which relates gas flow rate, average pressure within drainage area and stabilized sand face flowing pressure developed by Houpeurt is commonly used for interpretation of conventional multi-rate gas well deliverability tests and its above mentioned versions. The Houpeurt equation is the theoretical one contrary to Rawlins-Shellhard formula which is an empirical equation. The Houpeurt theoretical equation which is generally used for high flow rate wells has the following form:

$$
p_{s}^{2}-p_{b h f p}^{2}=a Q+b Q^{2}
$$

where:

$p_{s}$ - average pressure within drainage area of the well or initial flowing pressure,

$p_{b h f p}-$ stabilized bottom hole flowing pressure (stabilized sand face flowing pressure),

$Q$ - stabilized flow rate, $a, b$ - coefficients.

If $a$ and $b$ are known the absolute open flow potential $Q_{a b s}$ can be evaluated and the inflow performance curve can be generated, enabling calculation of the sand face drawn down pressure needed to produce the required gas flowing rate. The problems related to execution and interpretation of the conventional gas well deliverability tests, are widely discussed in literature and well known to petroleum engineers and thus there is no reason to discuss them here.

\section{Modified concept for carrying out and interpretation of multi-rate gas well test}

In practice Eq. 1 doesn't always precisely describe the relation between gas flow rate $Q$, stabilized sand face pressure, and average pressure within the drainage area of the well being tested. This is mostly caused by a phenomenon occurring within the well bore zone such as:

- precipitation of gas condensates due to pressure and temperature drop,

- difference between permeability of the well bore zone and reservoir,

- installation of the sand control screens,

- well completion (perforation or open hole completion).

One should also recall that several simplifying assumptions were made in derivation of Eq. 1 which are not satisfied in a real world scenario, which causes that sometimes the data of one or more flowing cycles must be rejected if they drift away from the linear trend of $\left(p_{s}^{2}-p_{b h f p}{ }^{2}\right) / Q$ vs. $Q$ or when the correlation of data is poor. If this happens the authors propose to modify the segment describing the impact of flow turbulence and use a slightly different form of Eq. 1, hoping that it would improve the flexibility and accuracy of the interpretation.

$$
p_{s}^{2}-p_{b h f p}^{2}=a Q+b Q^{n}
$$

There are three unknowns $a, b$ and $n$ because $n$ is allowed to be different than 2 if measurements indicate so. The calculation of $Q_{a b s}$ and construction of the IPR curve using standard procedure used in case of Eq. 1 (i.e. finding $a, b$ and $n$ using the least squares method) is extremely inconvenient mathematically. If Eq. 2 is to be used the following procedure is recommended for the conduction and interpretation of multi-rate gas well deliverability tests. Let's assume that the flow rates are consecutively growing for each succeeding flow cycle.
1. Start flowing the well with the first flow rate $Q_{1}$ and record the corresponding stabilized sand face flowing pressure $p_{b h f p 1}$.

2. Shut in the well for pressure stabilization to original average pressure within drainage area $p_{s}$.

3. Repeat procedure indicated in steps 1) and 2) for $Q_{N}$ where $Q_{N}$ is some maximum flow rate planned.

4. Calculate the flow rates of intermediate flow cycles using the following formula.

$$
Q_{N-i}=Q_{1}^{\frac{i}{N-1}} Q_{N}\left(1-\frac{i}{N-1}\right)
$$

where $i=1,2, \ldots, N-2 ; N$ - number of flow cycles.

5. Carry out the series of flow cycles with intermediate flow rates and record the stabilized sand face flowing pressures corresponding to each flow rate. Each flow cycle should be followed by a pressure build up period to original average reservoir pressure $p_{s}$.

6. Calculate $C_{i}$ for all flow rates:

$$
C_{1}-\left(p_{s}^{2}-p_{b h f p i}^{2}\right) / Q_{i}
$$

where $i=1,2, \ldots, N$.

7. Calculate $a$ using the following formula:

$$
a=\frac{1}{N-2} \sum_{i=1}^{N-2}\left(\frac{C_{i} C_{i+2}-C_{i+1}^{2}}{C_{i}+C_{i+2}-2 C_{i+1}}\right)
$$

where $i=1,2, \ldots, N-2$.

A) Present the Eq. 1 in a following form:

$$
\log \left(C_{i}-a\right)=(n-1) \log Q_{i}+\log b
$$

Mark the $\log \left(C_{i}-a\right)$ vs. $\log Q_{i}$ on rectangular coordinates. The values of $\log \left(C_{i}-a\right)$ vs. $\log Q_{i}$ should plot along 
the straight line - with slope $(n-1)$ enabling calculation of $n$ - which intersect the ordinate axis in $m=\log b$ for $Q=1$ enabling calculation of $b$.

The $n$ and $b$ values may be also calculated using the following formulas:

$$
n=1+\frac{N \sum_{i=1}^{N} \log \left(C_{i}-a\right) \log Q_{i}-\sum_{i=1}^{N} \log \left(C_{i}-a\right) \sum_{i=1}^{N} \log Q_{i}}{N \sum_{i=1}^{N}\left(\log Q_{i}\right)^{2}-\left(\sum_{i=1}^{N} \log Q_{i}\right)^{2}}
$$

$\log b=\frac{\sum_{i=1}^{N} \log \left(C_{i}-a\right) \sum_{i=1}^{N}\left(\log Q_{i}\right)^{2}-\sum_{i=1}^{N} \log \left(C_{i}-a\right) \log Q_{i} \sum_{i=1}^{N} \log Q_{i}}{N \sum_{i=1}^{N}\left(\log Q_{i}\right)^{2}-\left(\sum_{i=1}^{N} \log Q_{i}\right)^{2}}$

where $i=1,2, \ldots, N$.

or

B) Calculate $n$ using the following formula

$$
n=1+\frac{1}{N-1} \sum_{i=1}^{N-1} \frac{\log \frac{C_{i}-a}{C_{i+1}-a}}{\log \frac{Q_{i}}{Q_{i+1}}}
$$

where $i=1,2, \ldots, N-1$.

Knowing $n$ calculate $b$ using the following formula

$$
b=\frac{1}{N} \sum_{i=1}^{N} \frac{C_{i}-a}{Q_{i}^{n-1}}
$$

The procedure shown above may also be applied when the gas pseudo pressures $m(p)$ are used instead of $p^{2}$ in Eq. 1 . This will only require some simple modification in Eq. 4.

Equation (2) is the more general form of the theoretical Eq. (1). If the $p_{b h f p_{i}}$ and $Q_{i}$ perfectly satisfy the theoretical equation $p_{s}^{2}-p_{b h f p_{i}}^{2}=a Q_{i}+b Q_{i}^{2}$ and $Q_{i}$ satisfies condition given by Eq. (3) then the $a, b$ and $n$ coefficients calculated using the herein presented approach and conventional method will be practically the same (i.e. $n$ will be equal 2 ), which can be easily demonstrated using simple calculations.

Indeed, let us consider Example 1 given below for which, $a$ and $b$ coefficients calculated using the conventional method are $a=0.5713 \mathrm{MPa}^{2} /\left(\mathrm{Nm}^{3} / \mathrm{min}\right)$ and $b=0.0013 \mathrm{MPa}^{2} /$ $\left(\mathrm{Nm}^{3} / \mathrm{min}\right)^{2}$. The flow rates $Q_{i}$ which satisfy Eq. 3 and corresponding theoretical values of $p_{b h f p_{i}}$ (calculated using formula $p_{b h f p_{i}}=\sqrt{\left.p_{s}{ }^{2}-0.5713 Q_{i}-0.0013 Q_{i}^{2}\right)}$ are given in columns 1 and 2 respectively. The auxiliary coefficient $C_{i}$ (Eq. 4$)$ is given in column 3 of the Table 1 below.

Table 1.

\begin{tabular}{|c|c|c|c|}
\hline $\mathrm{N}$ & $\begin{array}{c}Q_{\mathrm{i}} \\
{\left[\mathrm{Nm}^{3} / \mathrm{min}\right]}\end{array}$ & $\begin{array}{c}p_{\text {bhfpi }} \\
{[\mathrm{MPa}]}\end{array}$ & $\begin{array}{c}C_{i} \\
\mathrm{MPa}^{2} /\left(\mathrm{Nm}^{3} / \mathrm{min}\right)\end{array}$ \\
\hline 1 & 88.49 & 12.310 & 0.6865 \\
\hline 2 & 108.30 & 11.626 & 0.7120 \\
\hline 3 & 132.55 & 10.664 & 0.7436 \\
\hline 4 & 162.23 & 9.240 & 0.7823 \\
\hline
\end{tabular}

The coefficients $a, b$ and $n$ calculated using the proposed modified approach are as follows:

$$
a=\frac{1}{N-2} \sum_{i=1}^{N-2}\left(\frac{C_{i} C_{i+2}-C_{i+1}^{2}}{C_{i}+C_{i+2}-2 C_{i+1}}\right)=0.5713
$$

$$
\begin{gathered}
n=1+\frac{1}{N-1} \sum_{i=1}^{N-1} \frac{\log \frac{C_{i}-a}{C_{i+1}-a}}{\log \frac{Q_{i}}{Q_{i=1}}}=2 \\
b=\frac{1}{N} \sum_{i=1}^{N} \frac{C_{i}-a}{Q_{i}^{n-1}}=0.0013
\end{gathered}
$$

i.e. they are the same as those calculated using the conventional method. The interpretation of multi-rate flow tests using the conventional method, means the adaptation of measurements "by force" to theory, while the proposed modified approach seems to be more flexible and better reflects the bottom hole pressure vs. flow rate relation.

\section{Examples of interpretation}

The proposed multi-rate gas well deliverability tests have never been used before, but among hundreds of test samples the authors succeeded in finding a dozen or so, for which flow rates in intermediate cycles were coincidentally almost equal to that indicated by Eq. 3. In the Tables below in the column entitled "gas flow rate" the flow rates recorded are followed by flow rates required by Eq. 3 provided in brackets. The fact that multiple datasets which satisfied the flow rate requirements given by Eq. 3 yielded reasonable results, support the usefulness of the present approach. 


\section{Example no 1}

Well X1 (data from Theory and practice of the testing of gas wells. Calgary 1975, Chapter 3, page 25). Number of flow cycles $N=4$.

Average pressure within drainage area $p_{s}=14.57 \mathrm{MPa}$ (corrected value).

Table 2.

\begin{tabular}{|c|c|c|}
\hline $\begin{array}{c}\text { Number } \\
\text { of cycle }\end{array}$ & $\begin{array}{c}\text { Gas flow rate } Q_{\mathrm{i}} \\
{\left[\mathrm{Nm}^{3} / \mathrm{min}\right]}\end{array}$ & $\begin{array}{c}\text { Stabilized sand face pressure } p_{\text {bhfpi }} \\
{[\mathrm{MPa}]}\end{array}$ \\
\hline 1 & 88.49 & 12.300 \\
\hline 2 & $110.12\left(108.30^{*}\right)$ & 11.583 \\
\hline 3 & $134.70\left(132.55^{*}\right)$ & 10.659 \\
\hline 4 & 162.23 & 9.217 \\
\hline
\end{tabular}

${ }^{*}$ Calculated using Eq. (3).

Table 3.

\begin{tabular}{|c|c|c|c|c|c|}
\hline & $\begin{array}{c}\text { Stabilized deliverability } \\
\text { coefficient }\end{array}$ & $\begin{array}{l}\text { Turbulence } \\
\text { coefficient }\end{array}$ & Exponent & $\begin{array}{l}\text { Absolute open } \\
\text { flow potential }\end{array}$ & \\
\hline & $a$ & $b$ & $n$ & $Q_{a b s}$ & $R^{2}$ \\
\hline & $\mathrm{MPa}^{2} /\left(\mathrm{Nm}^{3} / \mathrm{min}\right)$ & $\mathrm{MPa}^{2} /\left(\mathrm{Nm}^{3} / \mathrm{min}\right)^{\mathrm{n}}$ & & $\mathrm{Nm}^{3} / \mathrm{min}$ & \\
\hline Version A & 0.6230 & 0.0001 & 2.4365 & 238.56 & 3.1055 \\
\hline Version B & 0.6230 & 0.0001 & 2.4965 & 236.48 & 2.3251 \\
\hline Conventional method & 0.5713 & 0.0013 & 2 & 241.55 & 3.4966 \\
\hline
\end{tabular}

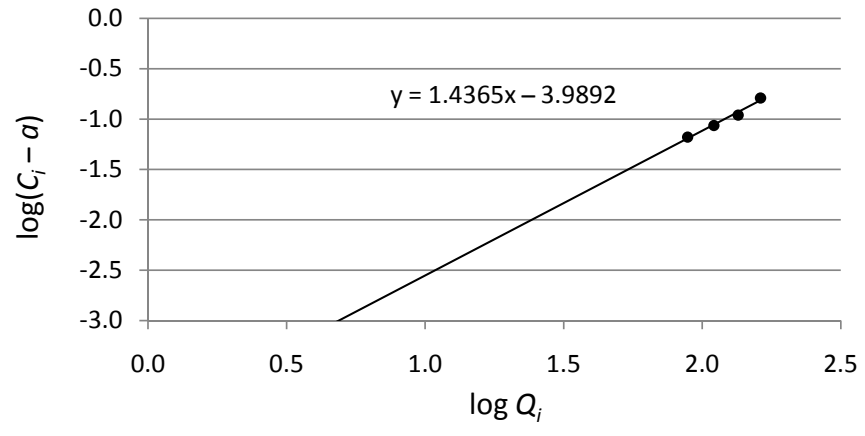

Fig. $1 . \log \left(C_{i}-a\right)$ vs. $\log Q_{i}$ curve

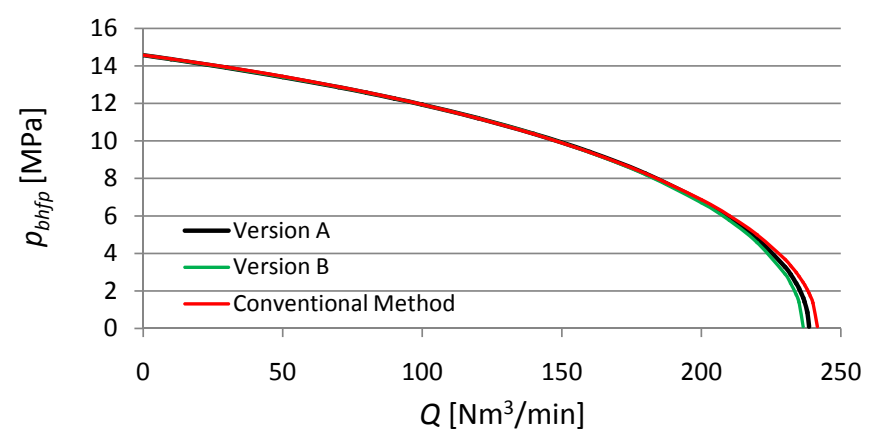

Fig. 2. Deliverability curves

\section{Example no. 2}

Well R-4.

Number of flow cycles $N=4$.

Average pressure within drainage area $p_{s}=25.98 \mathrm{MPa}$.
Table 4.

\begin{tabular}{|c|c|c|}
\hline $\begin{array}{c}\text { Number } \\
\text { of cycle }\end{array}$ & $\begin{array}{c}\text { Gas flow rate } Q_{\mathrm{i}} \\
{\left[\mathrm{Nm}^{3} / \mathrm{min}\right]}\end{array}$ & $\begin{array}{c}\text { Stabilized sand face pressure } p_{\text {bhfpi }} \\
{[\mathrm{MPa}]}\end{array}$ \\
\hline 1 & 77.20 & 25.82 \\
\hline 2 & $94.10\left(93.58^{*}\right)$ & 25.78 \\
\hline 3 & $115.60\left(113.43^{*}\right)$ & 25.72 \\
\hline 4 & 137.50 & 25.65 \\
\hline
\end{tabular}

* Calculated using Eq. (3).

Table 5.

\begin{tabular}{|c|c|c|c|c|c|}
\hline & $\begin{array}{c}\text { Stabilized deliverability } \\
\text { coefficient }\end{array}$ & $\begin{array}{l}\text { Turbulence } \\
\text { coefficient }\end{array}$ & Exponent & $\begin{array}{l}\text { Absolute open } \\
\text { flow potential }\end{array}$ & \\
\hline & $a$ & $b$ & $n$ & $Q_{a b s}$ & $R^{2}$ \\
\hline & $\mathrm{MPa}^{2} /\left(\mathrm{Nm}^{3} / \mathrm{min}\right)$ & $\mathrm{MPa}^{2} /\left(\mathrm{Nm}^{3} / \mathrm{min}\right)^{\mathrm{n}}$ & & $\mathrm{Nm}^{3} / \mathrm{min}$ & \\
\hline Version A & 0.0933 & 0.000036 & 1.5709 & 1111.42 & 0.0297 \\
\hline Version B & 0.0933 & 0.000037 & 1.5898 & 1119.21 & 0.0314 \\
\hline Conventional method & 0.0848 & 0.000280 & 2 & 1411.57 & 0.0346 \\
\hline
\end{tabular}




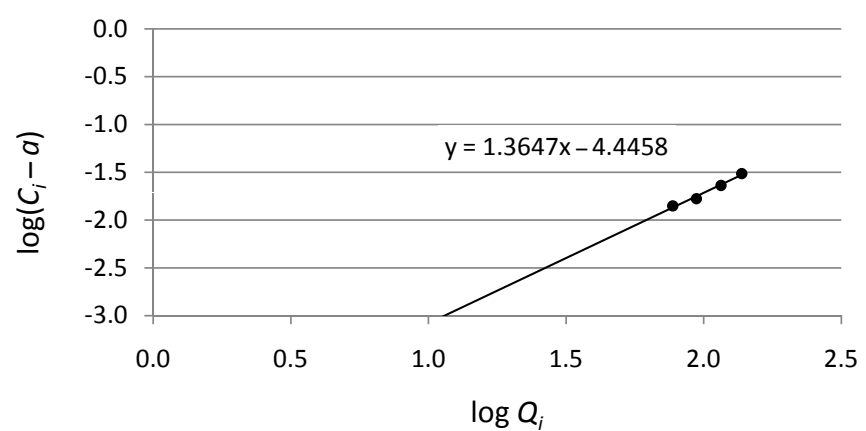

Fig. 3. $\log \left(C_{i}-a\right)$ vs. $\log Q_{i}$ curve

\section{Example no. 3}

Well K-2.

Number of flow cycles $N=4$.

Average pressure within drainage area $p_{s}=18.58 \mathrm{MPa}$.

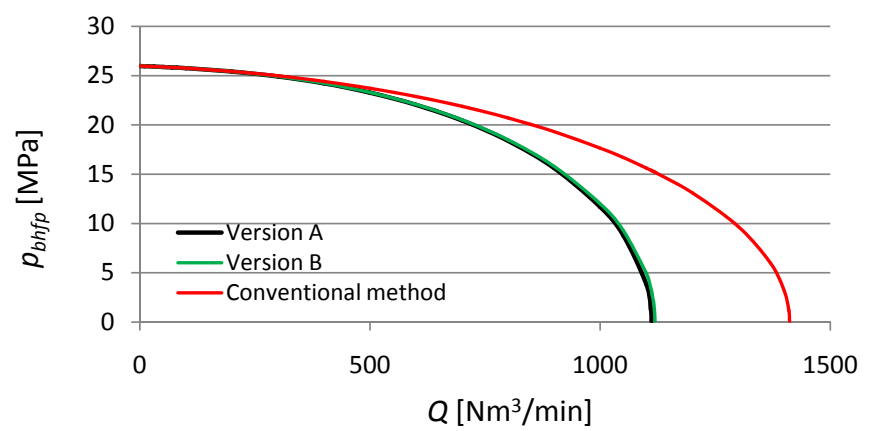

Fig. 4. Deliverability curves

Table 6.

\begin{tabular}{|c|c|c|}
\hline $\begin{array}{c}\text { Number } \\
\text { of cycle }\end{array}$ & $\begin{array}{c}\text { Gas flow rate } Q_{\mathrm{i}} \\
{\left[\mathrm{Nm}^{3} / \mathrm{min}\right]}\end{array}$ & $\begin{array}{c}\text { Stabilized sand face pressure } p_{\text {bhfpi }} \\
{[\mathrm{MPa}]}\end{array}$ \\
\hline 1 & 27.40 & 17.65 \\
\hline 2 & $42.40\left(41.17^{*}\right)$ & 17.00 \\
\hline 3 & $63.00\left(64.91^{*}\right)$ & 15.84 \\
\hline 4 & 99.90 & 13.14 \\
\hline
\end{tabular}

* Calculated using Eq. (3).

Table 7.

\begin{tabular}{|c|c|c|c|c|c|}
\hline & $\begin{array}{c}\text { Stabilized deliverability } \\
\text { coefficient }\end{array}$ & $\begin{array}{l}\text { Turbulence } \\
\text { coefficient }\end{array}$ & Exponent & $\begin{array}{l}\text { Absolute open } \\
\text { flow potential }\end{array}$ & \\
\hline & $a$ & $b$ & $n$ & $Q_{a b s}$ & $R^{2}$ \\
\hline & $\mathrm{MPa}^{2} /\left(\mathrm{Nm}^{3} / \mathrm{min}\right)$ & $\mathrm{MPa}^{2} /\left(\mathrm{Nm}^{3} / \mathrm{min}\right)^{\mathrm{n}}$ & {$[-]$} & $\mathrm{Nm}^{3} / \mathrm{min}$ & {$[-]$} \\
\hline Version A & 0.9686 & 0.0160 & 1.8388 & 163.14 & 0.9184 \\
\hline Version B & 0.9686 & 0.0165 & 1.8304 & 163.71 & 1.0341 \\
\hline Conventional method & 1.0414 & 0.0069 & 2 & 160.30 & 2.0431 \\
\hline
\end{tabular}

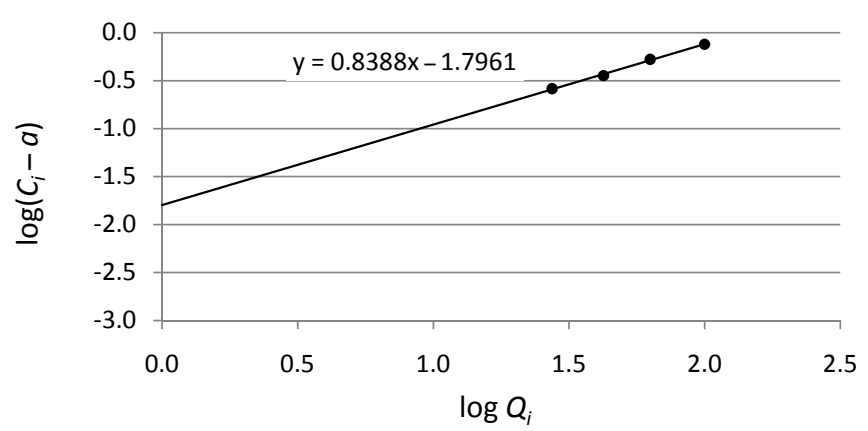

Fig. 5. $\log \left(C_{i}-a\right)$ vs. $\log Q_{i}$ curve

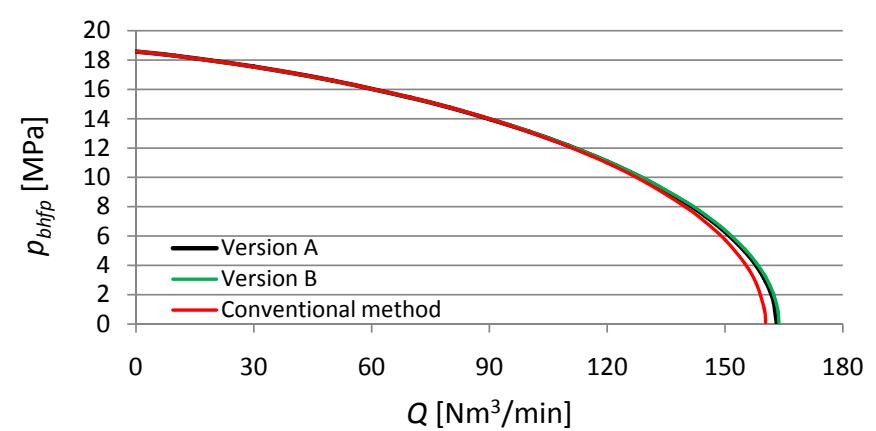

Fig. 6. Deliverability curves

\section{Example no. 4}

Well Z-7.

Number of flow cycles $N=4$.

Average pressure within drainage area $p_{s}=23.78 \mathrm{MPa}$

\begin{tabular}{|c|c|c|}
\hline $\begin{array}{c}\text { Number } \\
\text { of cycle }\end{array}$ & $\begin{array}{c}\text { Gas flow rate } Q_{\mathrm{i}} \\
{\left[\mathrm{Nm}^{3} / \mathrm{min}\right]}\end{array}$ & $\begin{array}{c}\text { Stabilized sand face pressure } p_{b h f p i} \\
{[\mathrm{MPa}]}\end{array}$ \\
\hline 1 & 16.80 & 23.06 \\
\hline 2 & $23.60\left(24.00^{*}\right)$ & 22.42 \\
\hline 3 & $34.80\left(35.30^{*}\right)$ & 21.16 \\
\hline 4 & 49.00 & 19.40 \\
\hline
\end{tabular}

${ }^{*}$ Calculated using Eq. (3). 
Table 9.

\begin{tabular}{|c|c|c|c|c|c|}
\hline & $\begin{array}{c}\text { Stabilized deliverability } \\
\text { coefficient }\end{array}$ & $\begin{array}{l}\text { Turbulence } \\
\text { coefficient }\end{array}$ & Exponent & $\begin{array}{l}\text { Absolute open } \\
\text { flow potential }\end{array}$ & \\
\hline & $a$ & $b$ & $n$ & $Q_{a b s}$ & $R^{2}$ \\
\hline & $\mathrm{MPa}^{2} /\left(\mathrm{Nm}^{3} / \mathrm{min}\right)$ & $\mathrm{MPa}^{2} /\left(\mathrm{Nm}^{3} / \mathrm{min}\right)^{\mathrm{n}}$ & {$[-]$} & $\mathrm{Nm}^{3} / \mathrm{min}$ & {$[-]$} \\
\hline Version A & 0,1524 & 0,3115 & 1,6471 & 93,73 & 88,8697 \\
\hline Version B & 0,1524 & 0,3116 & 1,6472 & 93,67 & 90,6460 \\
\hline Conventional method & 1,2280 & 0,0564 & 2 & 89,86 & 100,2032 \\
\hline
\end{tabular}

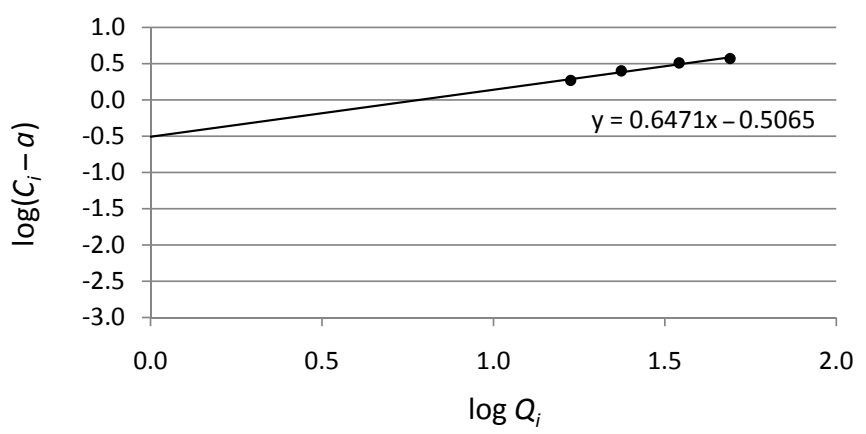

Fig. 7. $\log \left(C_{i}-a\right)$ vs. $\log Q_{i}$ curve

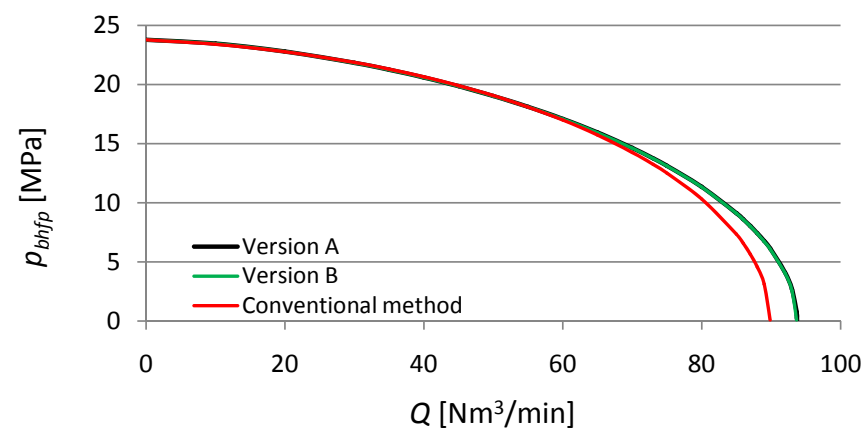

Fig. 8. Deliverability curves

\section{Example no. 5}

Well J-4.

Number of flow cycles $N=4$.

Average pressure within drainage area $p_{s}=25.613 \mathrm{MPa}$.

Table 10.

\begin{tabular}{|c|c|c|}
\hline $\begin{array}{c}\text { Number } \\
\text { of cycle }\end{array}$ & $\begin{array}{c}\text { Gas flow rate } Q_{\mathrm{i}} \\
{\left[\mathrm{Nm}^{3} / \mathrm{min}\right]}\end{array}$ & $\begin{array}{c}\text { Stabilized sand face pressure } p_{\text {bhfpi }} \\
{[\mathrm{MPa}]}\end{array}$ \\
\hline 1 & 45.90 & 25.601 \\
\hline 2 & $57.50\left(58.88^{*}\right)$ & 25.596 \\
\hline 3 & $75.50\left(75.54^{*}\right)$ & 25.587 \\
\hline 4 & 96.90 & 25.574 \\
\hline
\end{tabular}

${ }^{*}$ Calculated using Eq. (3).

Table 11

\begin{tabular}{|c|c|c|c|c|c|}
\cline { 2 - 6 } & \multirow{2}{*}{$\begin{array}{c}\text { Stabilized deliverability } \\
\text { coefficient }\end{array}$} & $\begin{array}{c}\text { Turbulence } \\
\text { coefficient }\end{array}$ & Exponent & $\begin{array}{c}\text { Absolute open } \\
\text { flow potential }\end{array}$ \\
\cline { 2 - 6 } & $a$ & $b$ & $n$ & $Q_{a b s}$ & $R^{2}$ \\
\cline { 2 - 6 } & $\mathrm{MPa}^{2} /\left(\mathrm{Nm}^{3} / \mathrm{min}\right)$ & $\mathrm{MPa}^{2} /\left(\mathrm{Nm}^{3} / \mathrm{min}^{\mathrm{n}}\right.$ & {$[-]$} & 2471.07 & 0.00001 \\
\hline Version A & 0.0057 & 0.00026 & 1.8826 & 2449.43 & 0.00002 \\
\hline Version B & 0.0057 & 0.00026 & 2133.74 & 0.00003 \\
\hline Conventional method & 0.0070 & 0.00014 & 2 & 213 & $\mathrm{Nm}^{3}$ \\
\hline
\end{tabular}

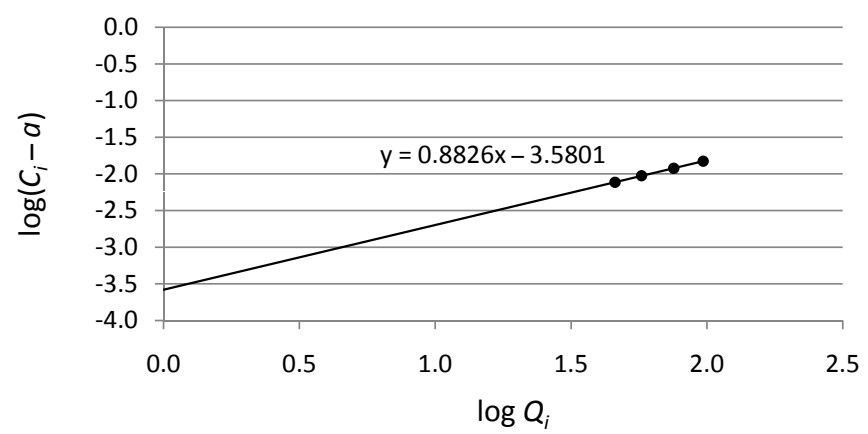

Fig. 9. $\log \left(C_{i}-a\right)$ vs. $\log Q_{i}$ curve

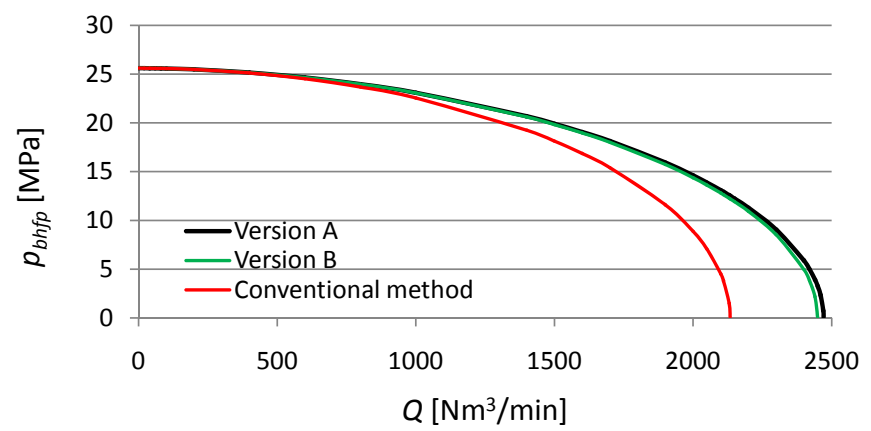

Fig. 10. Deliverability curves 


\section{Conclusions}

1. In the case of gas wells with low to moderate flow rates the difference between results attained using the conventional and the proposed method is usually small. The IPR curves sometimes nearly coincide, and absolute open flow potentials do not differ much between the conventional and proposed method.

2. For high flow rate wells analyzed, the difference between the results of the conventional and proposed method becomes significant.
3. The fact that multiple datasets which satisfy the flow rate requirements given by Eq. 3 yielded reasonable results seems to support the usefulness of the proposed procedure.

4. Above conclusions are based on limited data (dozen or so wells were analyzed which is rather insufficient) and so the proposed testing procedure should be verified using much more data, specifically from high flow rate wells which are unfortunately unavailable.

\section{Nomenclature}

$p_{s}$ - average pressure within drainage area of the well,

$p_{\text {bhfpi }}$ - stabilized bottom hole flowing pressure (sand face flowing pressure) for $i$-th flow rate,

$Q_{1}$ - flow rate of the first cycle,

$Q_{N}$ - flow rate of the $N$-th cycle,
$Q_{i}$ - flow rate of the $i$-th cycle,

$Q_{a b s}$ - absolute open flow potential,

$a, b, n$ - coefficients of Eq. 2 ,

$i$ - index,

$N$ - number of flow cycles.

Please cite as: Nafta-Gaz 2014, no. 1, pp. 15-22, DOI: 10.18668/NG2016.01.02

Article contributed to the Editor 7.09.2015. Approved for publication 1.10.2015.

The article is based on research entitled: The modified method of implementation testing gas wells with high efficiency using an adjustable choke - work Oil and Gas Institute - National Research Institute commissioned by PGNiG S.A., archive no.: DK4100-155/14, no. order: 989/SI/14.

\section{Literature}

[1] Dake L. P.: Fundamentals of reservoir engineering. Elsevier 1978.

[2] Energy Resources Conservation Board, Gas Well Testing. Calgary 1978, third edition.

[3] Houperurt A.: On the flow of gases in porous media. Revue de L'Institute Francais du Petrole 1959, 14, 11, pp. 1468-1684.

[4] Johns L. G., Blount E. M., Glaze O. H.: Use of the short term multiple rate flow test to predict performance of well having turbulence. Society of Petroleum Engineers, Annual Fall Technical Conference and Exhibition, New Orleans, Louisiana 1976. http://dx.doi.org/10.2118/6133-MS.

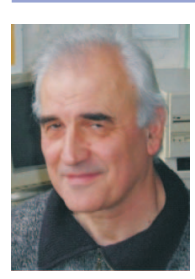

Dr. Eng. Tadeusz SZPUNAR PhD.

Assistant Professor

Department of Petroleum Engineering

Oil and Gas Institute - National Research Institute

ul. Lubicz 25 A

31-503 Kraków

E-mail: tadeusz.szpunar@inig.pl
[5] Johnson J. L., Lee W. J., Blasingname T. A.: Estimating the stabilized deliverability of a gas well using the Rawlings and Schelhardt method: An analytical approach. Society of Petroleum Engineers, Eastern Regional Meeting, Lexington, Kentucky 1991. http://dx.doi.org/10.2118/23440-MS.

[6] Lee J., Wattenbarger R. A.: Gas reservoir engineering. Society of Petroleum Engineers Textbook Series 1996, vol. 5.

[7] Szpunar T., Budak P.: Uwagi na temat metodyki interpretacji danych wielocyklowego testu przyplywu gazu do odwiertu. Nafta-Gaz 2007, no. 2, pp. 115-124.

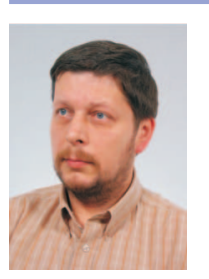

Paweł BUDAK M.Sc. Eng.

Senior Science and Research Specialist

Head of the Department of Petroleum Engineering

Oil and Gas Institute - National Research Institute

ul. Lubicz 25 A

31-503 Kraków

E-mail:pawel.budak@inig.pl

\section{Appendix A}

If Eq. 2 is used for the interpretation of multi-rate gas well deliverability testing we can write for each stabilized flow rate $Q_{i}$

$$
p_{s}^{2}-p_{b h f p_{i}}^{2}=a Q_{i}+b Q_{i}^{n}
$$

where $i=1,2, \ldots, N ; N$ - number of flow cycles.
We can write basing on Eq. (A.1):

$$
\left(\frac{Q_{i}}{Q_{i+1}}\right)^{n-1}=\frac{C_{i}-a}{C_{i+1}-a}
$$

where $i=1,2, \ldots, N-1$ and 


$$
C_{i}=\frac{p_{s}{ }^{2}-p_{b h f p_{i}}{ }^{2}}{Q_{i}}
$$

We can see from (A.2) that if

$$
Q_{i+1}=\left(Q_{i} Q_{i+2}\right)^{\frac{1}{2}}
$$

than

$$
\frac{C_{i}-a}{C_{i+1}-a}=\frac{C_{i+1}-a}{C_{i+2}-a}
$$

where $i=1,2 ., \ldots, N-2 ; N-$ number of flow cycles.

Solving (A.5) for $a$ we have:

$$
a_{i}=\frac{C_{i} C_{i+2}-C_{i+1}^{2}}{C_{i}+C_{i+2}-2 C_{i+1}}
$$

On the right hand side of Eq. (A.6) there are measured values related to $i$-th, $i+1$ and $i+2$ flow cycles so we added index $i$ to $a$. The most reliable value of $a$ can be found as the minimum of $\sum_{i=1}^{N-2}\left(a-a_{i}\right)^{2}$ using the least squares method, i.e. calculating $\partial S / \partial a$ where

$$
S=\sum_{i=1}^{N-2}\left(a-\frac{C_{i} C_{i+2}-C_{i+1}^{2}}{C_{i}+C_{i+2}-2 C_{i+1}}\right)^{2}
$$

and so we have from (A.7)

$$
a=\frac{1}{N-2} \sum_{i=1}^{N-2}\left(\frac{C_{i} C_{i+2}-C_{i+1}^{2}}{C_{i}+C_{i+2}-2 C_{i+1}}\right)
$$

If Eq. (A.4) is satisfied the flow rates which condition the validity of Eq. (A.8) can be calculated using the following formula

$$
Q_{N-i}=Q_{1}^{\frac{i}{N-1}} Q_{N}\left(1-\frac{i}{N-1}\right)
$$

where $i=1,2, \ldots, N-2 ; N$ - number of flow cycles.

A) Knowing $a$ the Eq. (A.1) can be presented in the following form:

$$
\log \left(C_{i}-a\right)=(n-1) \log Q_{i}+\log b
$$

If the $\log \left(C_{i}-a\right)$ vs. $\log Q_{i}$ data points are marked on the rectangular coordinates they should plot along the straight line - with slope $(n-1)$ enabling calculation of $n$-which intersect the ordinate axis in $m=\log b$ for $Q=1$ enabling calculation of $b$.

The $n$ and $b$ values may be also calculated using the following formulas:

$$
n=1+\frac{N \sum_{i=1}^{N} \log \left(C_{i}-a\right) \log Q_{i}-\sum_{i=1}^{N} \log \left(C_{i}-a\right) \sum_{i=1}^{N} \log Q_{i}}{N \sum_{i=1}^{N}\left(\log Q_{i}\right)^{2}-\left(\sum_{i=1}^{N} \log Q_{i}\right)^{2}}
$$

and $\log b=\frac{\sum_{i=1}^{N} \log \left(C_{i}-a\right) \sum_{i=1}^{N}\left(\log Q_{i}\right)^{2}-\sum_{i=1}^{N} \log \left(C_{i}-a\right) \log Q_{i} \sum_{i=1}^{N} \log Q_{i}}{N \sum_{i=1}^{N}\left(\log Q_{i}\right)^{2}-\left(\sum_{i=1}^{N} \log Q_{i}\right)^{2}}$

Equations (A.11) and (A.12) were derived using the least squares method i.e. solving the system of two Equations $\frac{\partial K}{\partial n}=\frac{\partial K}{\partial m}=0$ where $m=\log b$ and

$$
K=\sum_{i=1}^{N}\left[\log \left(C_{i}-a\right)-(n-1) \log Q_{i}-m\right]^{2}
$$

B) If the requirements regarding gas flow rate of the series of gas flow cycles (Eq. (A.9)) enabling calculation of $a$ are satisfied the $n$ and $b$ values may be calculated analytically in the following way:

Eq. (A.2) can be presented in a following form:

$$
(n-1) \log \frac{Q_{i}}{Q_{i+1}}=\log \frac{C_{i}-a}{C_{i+1}-a}
$$

where $i=1,2, \ldots, N-1$, and so

$$
n_{i}=1+\frac{\log \frac{C_{i}-a}{C_{i+1}-a}}{\log \frac{Q_{i}}{Q_{i+1}}}
$$

On the right hand side of (A.15) there are measured values related to $i$-th and $i+1$ cycle of gas flow so we added index $i$ to the value of $n$. According to the least squares method the most reliable value of $n$ is defined by:

$$
n=1+\frac{1}{N-1} \sum_{i=1}^{N-1} \frac{\log \frac{C_{i}-a}{C_{i+1}-a}}{\log \frac{Q_{i}}{Q_{i+1}}}
$$

because it minimizes the sum

$$
\sum_{i=1}^{N-1}\left(n-n_{i}\right)^{2}
$$

Knowing value of $n$ one can calculate $b$ from Eq. (A.1)

$$
b_{i}=\frac{C_{i}-a}{Q_{i}^{n-1}}
$$

where $i=1,2, \ldots, N$.

According to the least squares method the most reliable value of $b$ is defined by:

$$
b=\frac{1}{N} \sum_{i=1}^{N} \frac{C_{i}-a}{Q_{1}^{n-1}}
$$

because (A.19) minimizes the sum

$$
\sum_{i=1}^{N}\left(b-b_{i}\right)^{2}
$$

